\title{
Tranexamic acid for surgical bleeding
}

\author{
Uncertainty over vascular occlusive events warrants an adequately powered RCT
}

\author{
Katharine Ker lecturer, lan Roberts professor of epidemiology \\ Clinical Trials Unit, London School of Hygiene \& Tropical Medicine, London, UK
}

Joint replacement accounts for a large share of the 230 million major operations carried out each year worldwide. Each year in England and Wales alone there are about 180000 hip and knee replacements. ${ }^{1}$ Bleeding is an important complication, and many patients require a blood transfusion. One strategy to reduce surgical bleeding is to use the antifibrinolytic drug tranexamic acid.

Tranexamic acid inhibits clot breakdown by reducing the binding of plasminogen to fibrin. A recent systematic review and meta-analysis of randomised controlled trials showed that tranexamic acid reduces surgical bleeding and blood transfusion by about one third. ${ }^{2}$ However, the effect of the antifibrinolytic on the risk of vascular occlusive events remains uncertain. Perioperative myocardial infarction often goes undetected as many patients do not experience ischaemic symptoms. ${ }^{3}$ Nevertheless, an increased cardiac troponin level, a sensitive marker of myocardial injury, is common after major surgery and is associated with appreciable morbidity and mortality. ${ }^{4}$

In a linked article (doi:10.1136/bmj.g4829), the effort by Poeran and colleagues to resolve the uncertainty about the effect of tranexamic acid on vascular occlusive events is therefore welcomed. ${ }^{5}$ In a retrospective cohort study the authors compared the outcomes of 20051 patients who received tranexamic acid during hip or knee arthroplasty with 852365 patients who did not, using data from 510 US hospitals. They found that tranexamic acid reduces blood transfusion with no increase in vascular occlusive events, even after adjusting for patient characteristics and comorbidities.

Can we now conclude that tranexamic acid is safe for routine use in joint replacement surgery? Giving a drug that reduces blood clot breakdown to patients at increased risk of vascular occlusive events must surely be based on reliable evidence. Although any increase or decrease in risk is likely to be moderate, because of the hundreds of thousands of older adults who undergo joint replacement surgery each year even a moderate effect could be important.

Reliable assessment of moderate effects requires strict control of random error and bias. ${ }^{6}$ Does the study by Poeran and colleagues fulfil these criteria?
Control of random error means big studies with large numbers of outcome events, and with over 872000 patients Poeran and colleagues' study is certainly big. However, rigorous control of bias means proper randomisation and here the study falls short. As the authors acknowledge, unmeasured or residual confounding is a large threat to validity that can be dealt with only by randomisation. The authors' defence that trials have limited "generalizability to more general populations" confuses statistical and scientific inference. Whereas statistical inference, the process of using sample information to make inferences about the population from which it was drawn, is helped by having a representative sample, scientific inference involves making valid conclusions about biology. ${ }^{7}$

Valuable insights into the effects of drugs can be obtained even from patients who are unrepresentative of those in whom the drug will be used. The finding of the Clinical Randomisation of an Antifibrinolytic in Significant Haemorrhage (CRASH-2) trial that tranexamic acid reduces death from bleeding in adults with trauma was generalised to children, with an adult dose recommended in children over 12 years and a weight related dose for younger children, even though this age group was explicitly excluded from the trial. ${ }^{8}$ But is there any good biological reason why tranexamic acid would work in a 20 year old and not a in 12 year old? Generalisability is not "established" by data from "actual everyday practice" but depends on valid information about treatment effects and informed judgment about the factors that may be relevant to the drug's mechanism of action.?

Treatments that reduce bleeding often increase the risk of thrombosis. According to the summary of product characteristics, ${ }^{9}$ tranexamic acid is contraindicated in patients with a history of arterial or venous thrombosis. However, in the CRASH-2 trial there were fewer vascular occlusive deaths associated with tranexamic acid and a statistically significant reduction in fatal and non-fatal myocardial infarction. ${ }^{10}$ The pathogenesis of perioperative myocardial infarction includes an imbalance in myocardial oxygen supply and demand, rupture of coronary artery plaque, and platelet activation. By reducing surgical bleeding, tranexamic acid might reduce the perioperative tachycardia that increases myocardial oxygen demand and prevent the decrease in haemoglobin level 
responsible for reduced myocardial oxygen supply. ${ }^{11}$

Furthermore, plasmin is a potent mediator of inflammation and by inhibiting plasmin production, tranexamic acid might reduce the inflammatory response thought to contribute to rupture of coronary artery plaque. ${ }^{11}$

If tranexamic acid reduces rather than increases the risk of myocardial infarction in patients requiring surgery, it could be a highly cost effective way to improve surgical safety. ${ }^{11}$ The large reduction in the risk of myocardial infarction reported by Poeran and colleagues is consistent with this hypothesis. Although it would be premature to recommend the routine use of tranexamic acid in general and orthopaedic surgery, it is surely time to resolve this uncertainty in an adequately powered randomised controlled trial.

Competing interests: We have read and understood BMJ policy on declaration of interests and declare the following interests: IR and KK are investigators on several randomised controlled trials of tranexamic acid in bleeding patients and have grant applications pending for trials of tranexamic acid in head injury and elective surgery although not orthopaedic surgery.

Provenance and peer review: Commissioned; not externally peer reviewed.
1 National Joint Registry for England \& Wales. 10th annual report, 2013. www.njrcentre. org.uk/nircentre/Reports.PublicationsandMinutes/Annualreports/tabid/86/Default.aspx. Ker K, Edwards P, Perel P, Shakur H, Roberts I. Effect of tranexamic acid on surgical bleeding: systematic review and cumulative meta-analysis. BMJ 2012;344:e3054. 3 Thygesen K, Alpert JS, Jaffe AS, Simoons ML, Chaitman BR, White HD, et al. Third universal definition of myocardial infarction. J Am Coll Cardiol 2012;60:1581-98.

4 VISION Study Investigators. Myocardial injury after noncardiac surgery: a large international prospective cohort study establishing diagnostic criteria, characteristics, predictors, and 30-day outcomes. Anesthesiology 2014;120:564-78.

5 Poeran J, Rasul R, Suzuki S, Danninger T, Mazumdar M, Opperer M, et al. Tranexamic acid use and postoperative outcomes in patients undergoing total hip or knee arthroplasty in the United States: retrospective analysis of effectiveness and safety. BMJ 2014;349:g4829.

6 Collins R, MacMahon S. Reliable assessment of the effects of treatment on mortality and major morbidity, I: clinical trials. Lancet 2001;357:373-80.

7 Rothman KJ, Gallacher JE, Hatch EE. Why representativeness should be avoided. Int $J$ Epidemiol 2013;42:1012-4

8 Royal College of Paediatrics and Child Health. Major trauma and the use of tranexamic acid in children. Evidence statement, 2012. www.rcpch.ac.uk/system/files/protected/page/ 121112 TXA\%20evidence\%20statement final\%20v2.pdf.

9 Cyklokapron Injection. Summary of product characteristics. Updated 29 Nov 2012

10 CRASH-2 trial collaborators, Shakur H, Roberts I, Bautista R, Caballero J, Coats T, et al. Effects of tranexamic acid on death, vascular occlusive events, and blood transfusion in trauma patients with significant haemorrhage (CRASH-2): a randomised, placebo-controlled trial. Lancet 2010;376:23-32.

11 Roberts I. Scientific letter: could tranexamic acid use in surgery reduce perioperative myocardial infarction? Heart 2013;99:1785.

Cite this as: BMJ 2014;349:94934

(c) BMJ Publishing Group Ltd 2014 